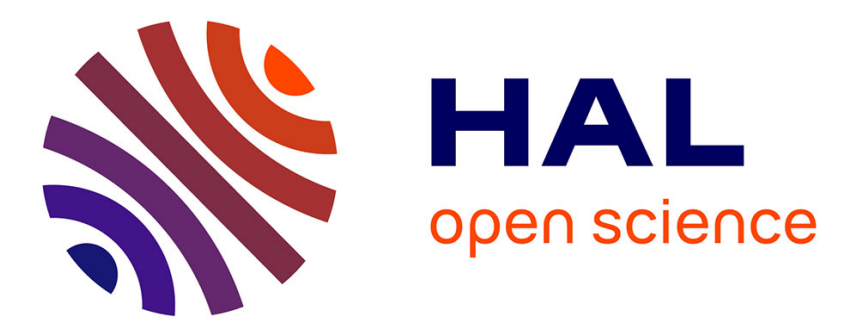

\title{
Decomposition of time-resolved tomographic PIV
}

\author{
Peter J. Schmid, D. Violato, O. Pust, F. Scarano
}

\section{To cite this version:}

Peter J. Schmid, D. Violato, O. Pust, F. Scarano. Decomposition of time-resolved tomographic PIV. 15th International Symposium on Applications of Laser Techniques in Fluid Mechanics, Jul 2010, Lisbonne, Portugal. pp.3.7.1. hal-01052852

\section{HAL Id: hal-01052852 \\ https://hal-polytechnique.archives-ouvertes.fr/hal-01052852}

Submitted on 28 Jul 2014

HAL is a multi-disciplinary open access archive for the deposit and dissemination of scientific research documents, whether they are published or not. The documents may come from teaching and research institutions in France or abroad, or from public or private research centers.
L'archive ouverte pluridisciplinaire HAL, est destinée au dépôt et à la diffusion de documents scientifiques de niveau recherche, publiés ou non, émanant des établissements d'enseignement et de recherche français ou étrangers, des laboratoires publics ou privés. 
15th INT SYMP ON APPLICATIONS OF LASER TECHNIQUES TO FLUID MECHANICS

Lisbon, Portugal, 05-08 July, 2010

\title{
Decomposition of time-resolved tomographic PIV
}

\author{
Peter J. Schmid ${ }^{1}$, Daniele Violato ${ }^{2}$, Oliver Pust ${ }^{3}$ and Fulvio Scarano ${ }^{2}$ \\ ${ }^{1}$ Laboratoire d'Hydrodynamique (LadHyX), CNRS-Ecole Polytechnique, F-91128 \\ Palaiseau, FRANCE \\ 2 Department of Aerospace Engineering, Delft University of Technology, 2600GB Delft, \\ THE NETHERLANDS \\ ${ }^{3}$ Dantec Dynamics, DK-2740 Skovlunde, DENMARK
}

\begin{abstract}
An experimental study has been conducted on a transitional water jet at a Reynolds number of $R e=5000$. Flow fields have been obtained by means of time-resolved tomographic particle image velocimetry (TR-TOMO PIV) capturing all relevant spatial and temporal scales. The measured three-dimensional flow fields have then been postprocessed by the dynamic mode decomposition (DMD) which identifies coherent structures that contribute significantly to the dynamics of the jet. Where the jet exhibits a primary axisymmetric instability followed by a pairing of the vortex rings, dominant dynamic modes have been extracted together with their amplitude distribution. These modes represent a basis for the low-dimensional description of the dominant flow features.
\end{abstract}

\section{Introduction}

The description of dominant and coherent flow features and their extraction from experimental data is the goal of many scientific studies of fluid flow. Dominant coherent structures are defined as organized fluid elements that capture the overall dynamics of the flow and are responsible for the bulk of mass, momentum and energy transfer. Despite this attempt to describe coherence in fluid flow, no definitive consensus has been reached, and various notions, mostly based on statistical means, are in common use. Descriptions by probability density functions as well as spatial covariances are among the more popular and successful classifications of fluid elements and the importance of their role in the overall flow dynamics.

As varied as the definition of coherence is the range of numerical algorithms to extract pertinent information from the flow. In experimental settings, conditional averaging (biasing statistics towards specific events in the flow) as well as quadrant analysis (evaluating the occurrence and frequency of specific sign-configurations in the velocity fields) were among the early techniques to explore recurring or persistent features of the flow. A less subjective techniques is based on the spatial 
correlation tensor of the flow whose eigenvalues decompose the flow into mutually decorrelated structures. This technique, known as the proper orthogonal decomposition (POD), reorders the flow into a hierarchy of energy-weighted structures which optimally capture the total kinetic energy of the flow when used as a Galerkin basis. It still enjoys great popularity among experimental and computational fluid dynamicists which is due to its versatility, its ease of implementation and its convergence properties based on an energy norm.

Computational fluid dynamicists faced the same issues of coherent feature extraction when analyzing the flow fields computed by direct numerical simulations or other techniques. The wealth of data generated by simulations had to be postprocessed to delineate the important dynamic structures from the incoherent featureless noise. In contrast to experimentalists, however, they could rely on a set of model equations that built the foundation of their simulations, and efficient algorithms could be developed that exploited this fact. Among these algorithms, the Arnoldi method and its variants dominate the quantitative analysis of fluid flow. The Arnoldi method, an iterative Krylov subspace technique to compute eigenvalues of large-scale matrices, has rapidly become a standard tool to compute stability information of flows in complex geometries. When coupled with numerical simulations it produces global stability modes together with their frequency and growth/decay rates. Various modifications have been developed over the years to improve overall performance, to direct convergence towards specific eigenvalues and to add robustness. Central to the algorithm is the construction of an orthogonal set of vectors (flow fields) onto which the dynamics is projected. This construction depends on the availability of model information, as it requires the evaluation of the underlying equations using a given flow field. While this algorithmic step is easily accomplished by numericists, it constitutes an obstacle for a straightforward application to experimentally generated flow field data. For this very reason, many iterative techniques that are routinely applied within a computational framework are not available to the experimentalists. It is thus fair to say, that the range of options for a quantitative analysis of experimental fluid data considerably lags behind the possibilities available to computational fluid dynamicists.

The past years have seen remarkable advances in experimental data-acquisition and image analysis, and flow data from experiments rival data from large-scale numerical simulations in spatial and temporal resolution as well as in complexity. The analysis of unsteady three-dimensional flow fields is no longer the domain of computational fluid dynamicist owing to the development of time-resolved tomographic PIV techniques [2]. Algorithms for the analysis of these data are now needed to allow the same depth of exploration that is customary in a computational setting. The dynamic mode decomposition (DMD) is such a technique as it is solely based on data and does not depend on access to an underlying set of equations. It is related to the Arnoldi method mentioned above but replaces the projection onto an orthogonal basis by a projection onto a snapshot sequence. In this manner, spectral information about the flow can be extracted from the measurements.

After describing the experimental setup and the principles of the dynamic mode decomposition, a set of time-resolved tomographic PIV-measurements of a water jet will be processed and analyzed. The obtained results will be presented in form of their spectral characteristics (frequencies, growth/decay rates, wavenumbers and amplitudes) and modal shapes. A discussion of the presented material and an outlook of future applications will conclude this article. 


\section{Experimental setup and data decomposition}

\subsection{Experimental setup}

The experiments have been performed in the water jet facility at the Aerodynamic Laboratories of the TU Delft [1]. The jet exits from a round nozzle of diameter $D=10 \mathrm{~mm}$ into an octogonal water tank of $600 \mathrm{~mm}$ diameter and $800 \mathrm{~mm}$ height whose Plexyglass sides allow full optical access to the illumination and tomographic imaging. For a Reynolds number of $R e=5000$ a jet exit velocity of $U=0.5 \mathrm{~m} / \mathrm{s}$ has been chosen. Neutrally buoyant polyamide particles (of $56 \mu \mathrm{m}$ diameter) together with a solid-state Nd:YAG laser provide light-scatter images that are recorded by the tomographic system consisting of four CMOS cameras. Image sequences are acquired by this system at a kilo-hertz rate over a three-dimensional measurement domain of $50 \mathrm{~mm} \times 50 \mathrm{~mm} \times 32 \mathrm{~mm}$. Three such domains (phase matched across the overlap volumes) cover an extent of $130 \mathrm{~mm}$ along the jet axis. Results from the domain closest to the jet nozzle will be reported below; results from the remaining two domains will be included in the full conference contribution. The volumetric light intensity is reconstructed using a volume-self-calibration procedure and a MART reconstruction algorithm. Three-dimensional velocity fields are then computed based on a spatial cross-correlation of two subsequent volumes with LaVision Davis 7.4, and data post-processing using a space-time regression with a $5 \mathrm{pt} \times 5 \mathrm{pt} \times 5 \mathrm{pt} \times$ $5 p t$ kernel reduces velocity fluctuations due to measurement or processing noise [2]. A representative snapshot from the experiment is shown in figure 2(a), visualized by velocity vectors in the axial center-plane.

\subsection{Principles of the dynamic mode decomposition}

The dynamic mode decomposition (DMD) is a data-based decomposition technique that identifies the dominant coherent motion in a flow field by constructing and subsequently analyzing an approximate linear mapping between time-resolved measurements [3,4]. Given a sequence of measured flow fields, denoted by $\mathbf{v}_{j}$ and separated by a constant time-interval $\Delta t$, i.e.,

$$
\mathbf{V}_{1}^{N}=\left\{\mathbf{v}_{1}, \mathbf{v}_{2}, \ldots, \mathbf{v}_{N}\right\}
$$

with $N$ as the total number of flow fields, we assume a linear mapping $\mathbf{A}_{\Delta t}$ between each of the snapshots (assumed to be constant over the snapshot sequence). We have $\mathbf{v}_{j+1}=\mathbf{A}_{\Delta t} \mathbf{v}_{j}$. Applying the mapping $\mathbf{A}_{\Delta t}$ to the entire sequence $\mathbf{V}_{1}^{N}$ results in

$$
\mathbf{A}_{\Delta t} \mathbf{V}_{1}^{N}=\mathbf{V}_{2}^{N+1}
$$

For a sufficiently long sequence of snapshots from an experiment, it appears reasonable to assume that the flow fields become linearly dependent. When this limit is reached, it is possible to express any further snapshots by a linear combination of the previous ones; mathematically, this amounts to

$$
\mathbf{A}_{\Delta t} \mathbf{V}_{1}^{N}=\mathbf{V}_{2}^{N+1} \approx \mathbf{V}_{1}^{N} \mathbf{S}_{\Delta t}
$$

where $\mathbf{S}_{\Delta t}$ contains the coefficients of the above-mentioned linear combination. In this last equation, the action of $\mathbf{A}_{\Delta t}$ on the snapshot sequence $\mathbf{V}_{1}^{N}$ has been approximated by a combination (expressed 
by $\mathbf{S}_{\Delta t}$ ) of the members of $\mathbf{V}_{1}^{N}$. Spectral information about the high-dimensional matrix $\mathbf{A}_{\Delta t}$ is thus contained in the matrix $\mathbf{S}_{\Delta t}$ which can be thought of as a projection of $\mathbf{A}_{\Delta t}$ onto the snapshot basis $\mathbf{V}_{1}^{N}$. This projection is reminiscent of the Arnoldi method where the original large-scale matrix is replaced by a lower-dimensional Hessenberg matrix whose eigenvalues approximate some of the eigenvalues of the original matrix. The orthogonalization step of the Arnoldi method, however, is absent.

The matrix $\mathbf{S}_{\Delta t}$ can be computed from the above equation by a least-squares approximation based on the two data sets $\mathbf{V}_{1}^{N}$ and $\mathbf{V}_{2}^{N+1}$. We obtain

$$
\mathbf{S}_{\Delta t}=\mathbf{R}^{-1} \mathbf{Q}^{H} \mathbf{V}_{2}^{N+1}
$$

where $\mathbf{Q}$ and $\mathbf{R}$ stand for the $\mathbf{Q R}$-decompostion of the data set $\mathbf{V}_{1}^{N}$, that is, $\mathbf{Q R}=\mathbf{V}_{1}^{N}$. The eigenvalues of $\mathbf{S}_{\Delta t}$ approximate some of the eigenvalues of $\mathbf{A}_{\Delta t}$ and the corresponding eigenvectors of $\mathbf{A}$ are determined by $\mathbf{V}_{1}^{N} \mathbf{w}$ where $\mathbf{w}$ is an eigenvector of $\mathbf{S}_{\Delta t}$. We will refer to the quantities $\mathbf{V}_{1}^{N} \mathbf{w}$ as the dynamic mode of the snapshot series. Due to the nature of the data sequence, the eigenvalues $\lambda$ of $\mathbf{S}_{\Delta t}$ describe the inter-snapshot dynamics. For a sufficiently long data sequence sampled from a nonlinear process (experiment), they approach the unit-disk and represent a neutrally stable, oscillatory process. We often map the eigenvalues $\lambda$ of $\mathbf{S}_{\Delta t}$ via the transform $\omega=\log (\lambda) / \Delta t$; unstable eigenvalues $\omega$ appear then in the right half-plane.

The reliance on data allows a great deal of flexibility for the dynamic mode decomposition. The inclusion of only parts of the measured flow field in the data sequence $\mathbf{V}_{1}^{N}$ enables the exploration of subdomains where localized instabilities or flow phenomena are expected or observed. In addition, images from high-speed cameras can be as straightforwardly processed as data from time-resolved PIV measurements; the data may even be of a composite nature, combining, for example, PIV-velocity measurements with time-synchronous acoustic pressure signals from a microphone array in typical aero-acoustic applications. Even more significantly, the alignment of the snapshots in time represents only one of many options. For example, the data fields $\mathbf{v}_{j}$ could represent measurements at spatial positions $x_{j}$ separated by $\Delta x$. By forming and processing this spatially aligned data sequence, the resulting matrix $\mathbf{S}_{\Delta x}$ will contain spectral information about the spatial evolution of the flow. For a more detailed description of DMD, the reader is referred to $[3,4]$.

The critical parameters of the dynamic mode decomposition are the length $N$ of the snapshot sequence and the (temporal or spatial) separation $\Delta t, \Delta x$ between consecutive snapshots. The former parameter can be determined by observing the residual of the least-squares step above. The latter parameter has to be chosen to approximately match the characteristic time/space scale of the fluid flow under investigation, while simultaneously complying with the Nyquist frequency criterion.

DMD represents an approximation of a time-resolved sequence from a nonlinear process by a linear mapping between the samples. Mathematically, it is related to a Koopman analysis of a nonlinear dynamical system; an application of Koopman analysis to fluid flows has recently been presented [5].

\section{Results}

A sequence of snapshots has been recorded at a sampling frequency of $1 \mathrm{kHz}$. Each flow field consists 
of $107 \times 62 \times 62$ three-dimensional velocity vectors. With $N=40$ snapshots in time, the full data array contains more than $16 \times 10^{6}$ entries for each of the three fluid velocity components. This array will be processed by the dynamic mode decomposition to extract coherent structures of dynamic relevance.

\subsection{Temporal analysis}

In a first step, a temporal analysis will be attempted. For this case, the flow fields at each of the forty time-step will be reshaped into the columns of a data matrix $\mathbf{V}_{1}^{40}$. A mapping between the snapshots (expressed in the snapshot basis) will then be computed following the procedure described above.

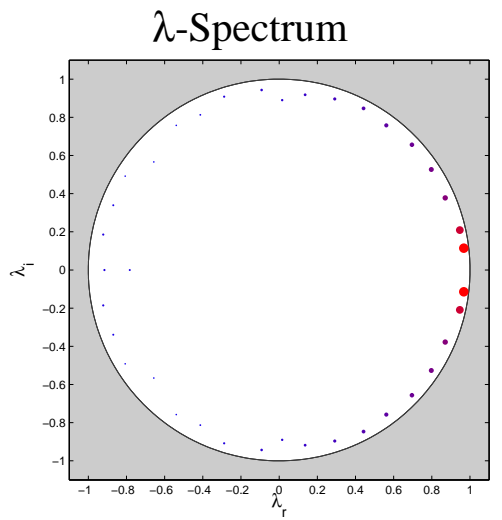

(a)

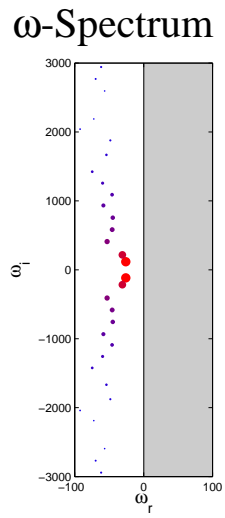

(b)
Amplitude

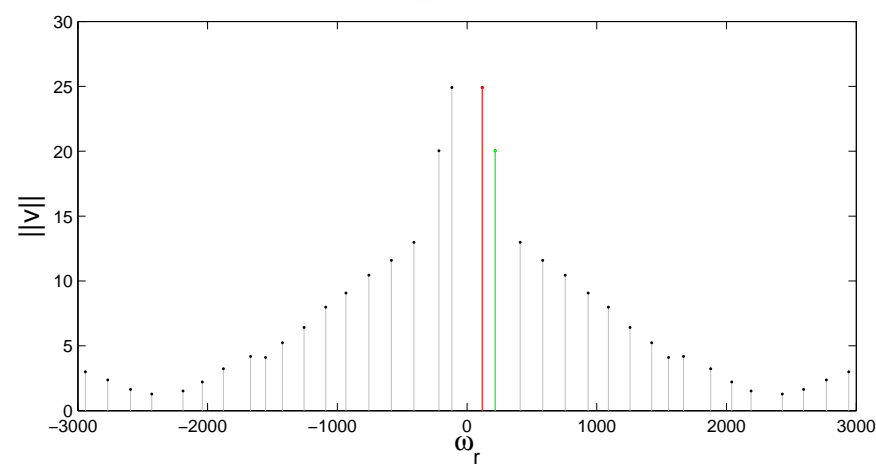

(c)

Figure 1: Decomposition of a three-dimensional low-Mach number jet at $R e=5000$ from time-resolved tomographic PIV measurements. (a) Eigenvalues of the matrix $\mathbf{S}_{\Delta t}$ representing the inter-snapshot dynamics. (b) Dynamic mode spectrum, logarithmically mapped (see text). (c) Amplitude distribution of the dynamic modes versus their temporal frequency $\omega_{r}$.

A $\mathbf{S}_{\Delta t}$-matrix of dimension $39 \times 39$ results whose eigenvalues $\lambda$ are shown in figure 1(a). An eigenvalue near $(1,0)$ signifying the mean flow (i.e. the temporally-averaged flow field of the data sequence) has been omitted in the figure. The size and color (from red to blue) of the eigenvalues indicate the amplitude of the respective structure in the data sequence. A transformed $\omega$-spectrum is shown in figure 1(b). In both spectra, we observe stable eigenvalues (inside the unit disk and in the left half-plane, respectively). The different scale of the imaginary and real axis in figure 1(b) should be noted which indicates the convergence of the eigenvalues towards a linear representation of a saturated nonlinear process. For a longer data-sequence, the eigenvalues are expected to tend towards the unit disk (in figure 1(a)) and the imaginary axis (in figure 1(b)). Due to real input data, the spectra are symmetric with respect to the real axis. A dominant mode (in red) is clearly visible whose Stouhal number, based on the jet diameter and the jet velocity, can be determined as $S t=0.374$. A second significant eigenvalue corresponds to a Strouhal number of $S t=0.671$. The amplitude distribution shown in figure 1(c) has been computed by projecting the data sequence onto the identified dynamic modes. The coefficients of this projection indicate the presence of specific dynamic modes in the original data sequence and thus determine their significance; again, the mean flow at $\omega_{r}=$ 0 has been omitted. A pronounced peak at two frequencies/Strouhal numbers can be observed. 


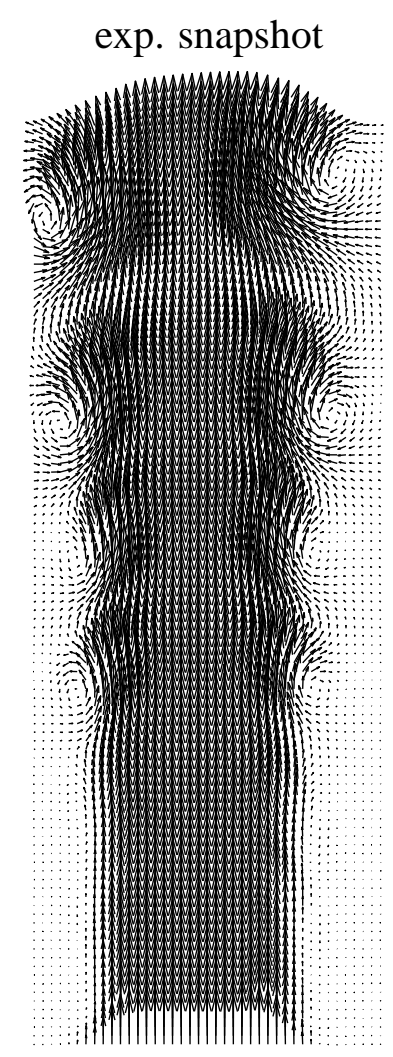

(a)

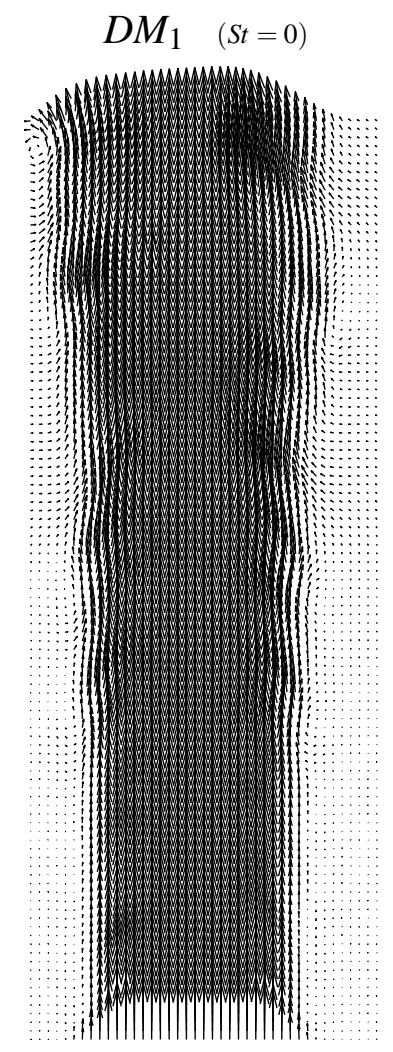

(b)

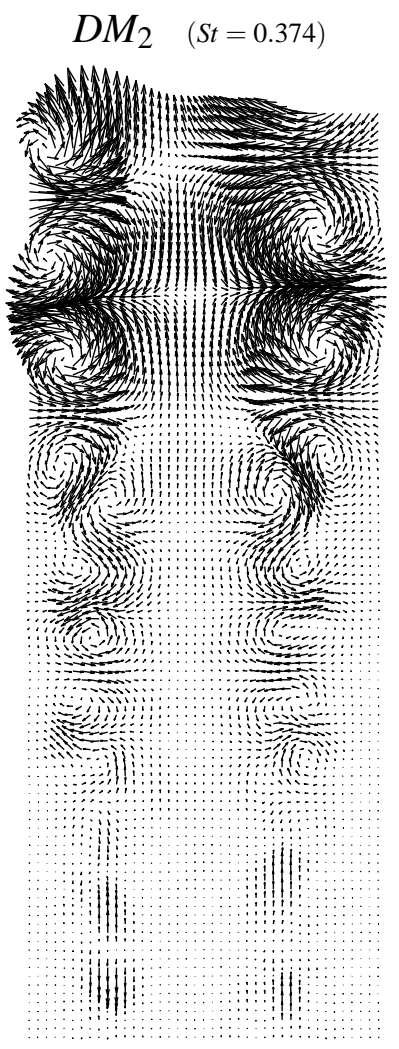

(c)

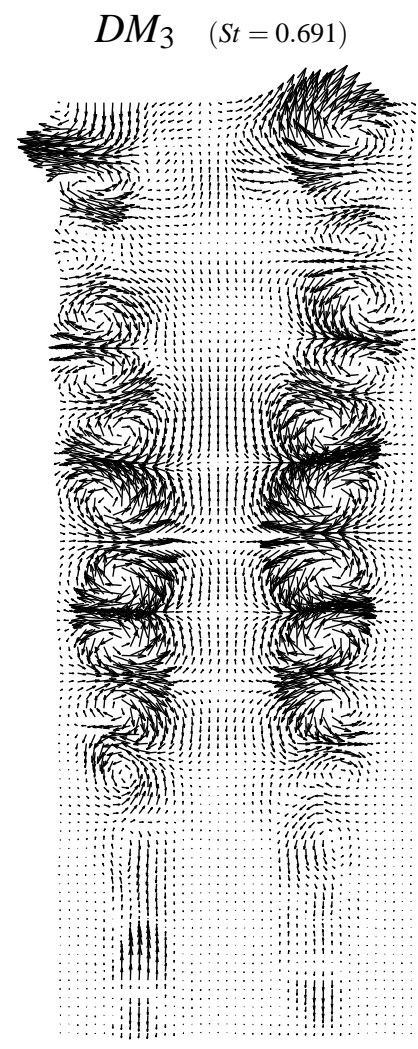

(d)

Figure 2: Decomposition of a three-dimensional low-Mach number jet at $R e=5000$. (a) Representative snapshot from the time-resolved tomographic PIV measurements. (b-d) Three most dominant dynamic modes (DM): mean flow (b) and two dynamic modes with a significant contribution in the original data sequence.

Higher-frequency modes contribute less and less to the data sequence, reflected in the decay of their respective amplitudes.

Figure 2 (b,c,d) shows the dynamic modes corresponding, respectively, to the mean flow and the two frequencies/Strouhal numbers indicated in red and green in the amplitude plot (figure 1(c)). All modes are visualized by velocity vectors in the axial center-plane. Small inhomogeneities near the edge of the jet are observed, which would gradually vanish once more snapshots were taken into account in the analysis. The next most dominant dynamic mode (indicated in red in the amplitude plot) is displayed in figure 2(c). It shows strong vortical structures near the edge of the jet about four diameters downstream from the nozzle, corresponding to vortex rings. The tendency toward an axisymmetric nature of the instability is clearly detectable and confirmed by a radial cut (not shown). The next-most dominant dynamic mode (indicated in green in the amplitude plot) is depicted in figure 2(d). It again features nearly axisymmetric, strong vortex rings, however, concentrated closer to the nozzle, with a reduced axial spacing and correspondingly higher Strouhal number $(S t=0.691)$. A superposition of the three displayed dynamic modes, each weighted by their temporal exponential 
dynamics $\exp (i \omega t)$ and initialized by a representative flow field, would capture the bulk of the jet dynamics and reproduce the principal features of the original data sequence.

The temporal dynamic mode decomposition has identified two distinct Strouhal numbers in the data sequence; the corresponding structures are characterized by nearly axisymmetric vortical structures superimposed on the cylindrical mean vortex sheet of the jet.

\subsection{Spatial analysis}

The previous analysis, detecting a periodic fluid motion with distinct frequencies, suggests to revisit the problem within a spatial framework. As mentioned previously, since the DMD does not depend on a particular model, a simple re-organization of the data array suffices to perform a spatial rather than a temporal analysis. To this end, we align the data fields in our matrix $\mathbf{V}_{1}^{N}$ in the axial direction, i.e., each column in $\mathbf{V}_{1}^{N}$ consists of a time-record of the three-dimensional flow field in the cross-sectional plane at a given axial location. The number of snapshots is accordingly $N=107$, and the computed matrix $\mathbf{S}_{\Delta x}$ is of size $106 \times 106$ and contains spatial spectral information. The time-coordinate becomes an independent variable of the resulting dynamic modes; consequently, the extracted spatial dynamic modes will contain a temporal dependency.

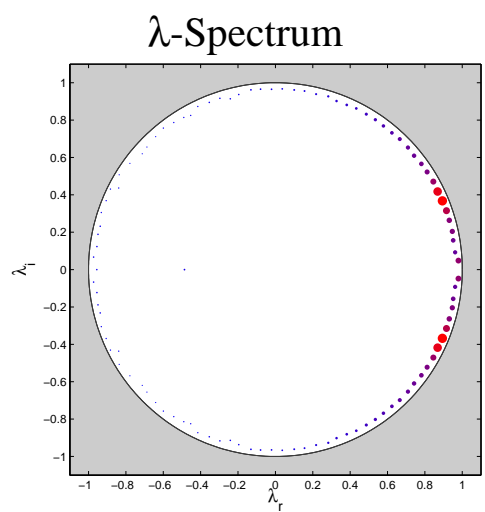

(a)

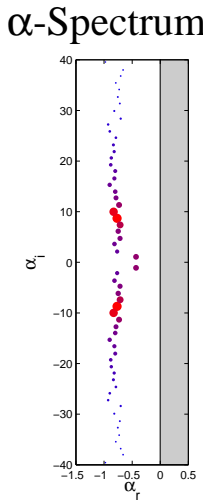

(b)

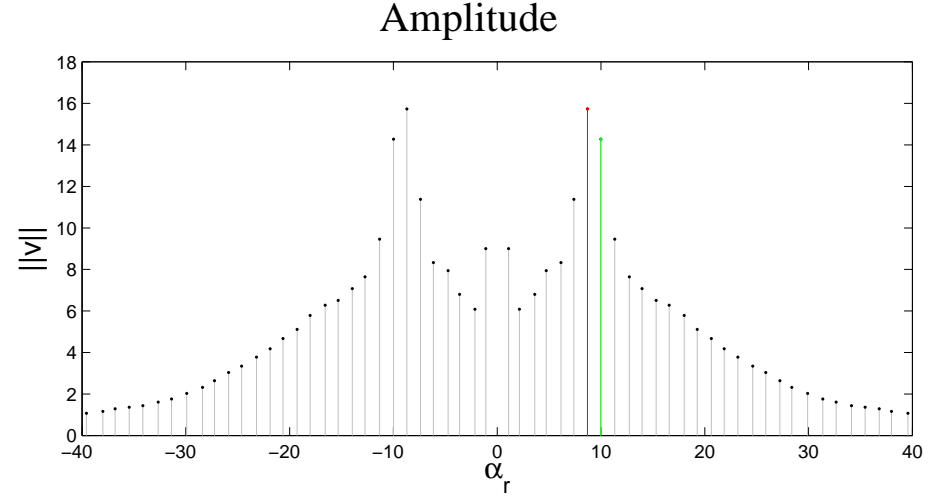

(c)

Figure 3: Spatial dynamic mode decomposition of a three-dimensional low-Mach number jet at $R e=5000$. (a) Spatial inter-snapshot spectrum, i.e., eigenvalues of $\mathbf{S}_{\Delta x}$. (b) spatial DMD-spectrum, logarithmically mapped (see text). (c) Amplitude distribution of the spatial dynamic modes versus their streamwise wavenumber $\alpha_{r}$.

Processing the spatially-aligned data matrix results in the spectra displayed in figure 3(a,b), again in the inter-snapshot format (figure 3(a)) and the more familiar mapped format according to $\alpha=$ $\log (\lambda) / \Delta x$. As in the temporal case, we notice a clustering of the eigenvalues near the unit disk and the neutral line, respectively. The "mean-flow eigenvalue" has been excluded as before. The spatial DMD detects a marked spatial wavenumber, indicated by the red eigenvalues in either spectrum. The importance and prevalence of this spatial structures is further confirmed in the amplitude distribution (see figure 3(c)) which identifies a peak near the spatial wavenumber $\alpha_{r} \approx 9$. On both sides of this peak, the amplitude of other detected wavenumbers decreases notably. The dynamic modes 


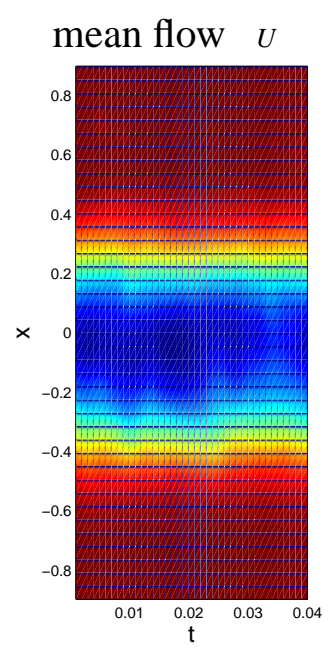

(a)

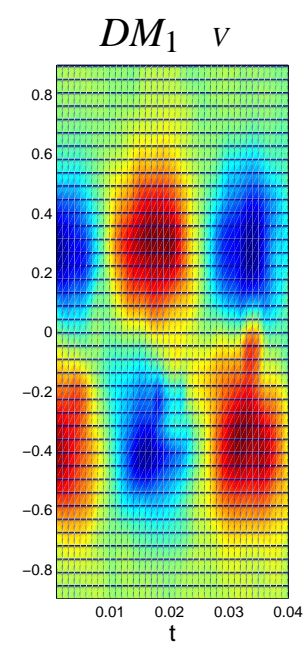

(b)

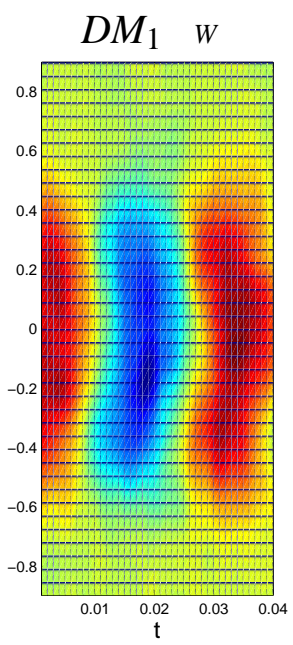

(c)

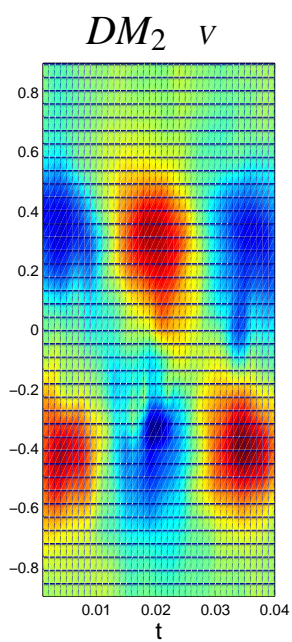

(d)

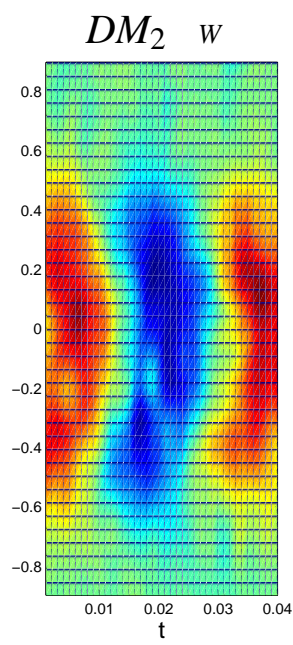

(e)

Figure 4: Spatial dynamic mode decomposition of a three-dimensional low-Mach number jet at $R e=$ 5000. (a) Spatial dynamic mode associated with the mean flow, visualized by the axial velocity. (b-c) First spatial dynamic mode, visualized by the velocity components in the cross-sectional plane. (d-e) Second spatial dynamic mode, visualized by the velocity components in the cross-sectional plane.

corresponding to the colored peaks in the amplitude distribution depend on the coordinates of the cross-sectional plane and on time (their streamwise dependence is given by $\exp (i \alpha x)$ with $\alpha$ as the respective eigenvalue) and are thus difficult to visualize. For this reason, we will first demonstrate the temporal dependence of the two dynamic modes identified in color in the amplitude distribution, evaluated in a one-dimensional cross-sectional cut through the center of the jet. The two modes and their temporal dependence are visualized by contours of one of the cross-sectional velocity component. In addition, the mean flow, visualized by the axial velocity component, is included for completeness (figure 4(a)); it show a steady velocity component in the center of the interrogation domain. The two displayed dynamic modes exhibit a clear temporal frequency in both velocity components. This should not come as a surprise as the temporal DMD analysis clearly extracted a well-defined Strouhal number from the data.

In a different visualization (figure 5), we display a temporal sequence of velocity vectors for the two dominant DMD-modes (after the mean flow mode). In both cases, we observe a circular motion in the cross-sectional plane which reverses direction over the course of the sampling period. This is consistent with the characteristics of the spatial dynamic modes depicted in figure 4(b-e). The final plot (in blue) represents the flow field associated with the most dominant Fourier mode of the temporal sequence. For a full appreciation of the three-dimensional dynamics of the dynamic modes, each spatial dynamic mode has to be augmented by an exponential/oscillatory evolution in the streamwise direction according to $\exp (\alpha x)$. Nevertheless, the temporal sequences in figure 5 give a first indication of the complexity of the fluid motion captured in the three-dimensional data sequence. 

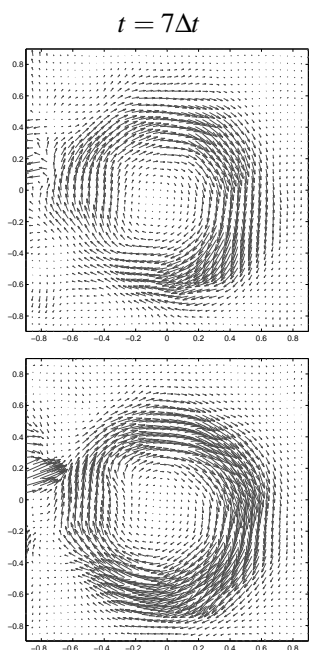

(a)
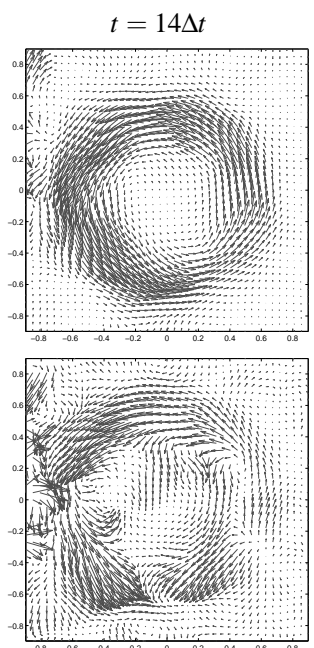

(b)
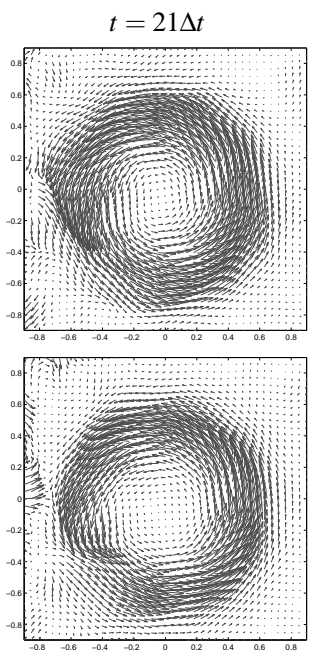

(c)
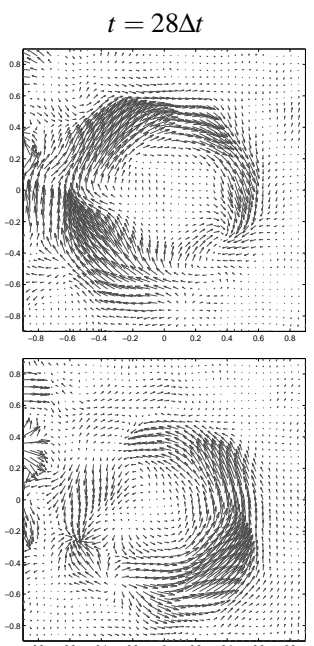

(d)
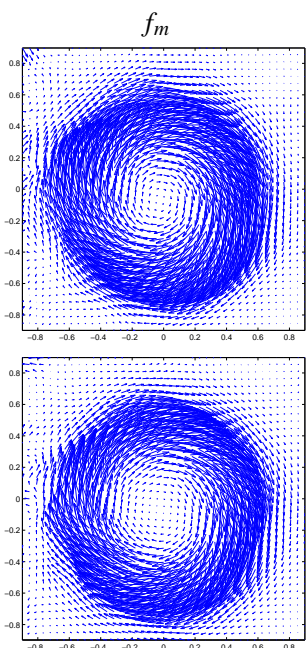

(e)

Figure 5: Decomposition of a three-dimensional low-Mach number jet at $R e=5000$. (a-d) Temporal sequence of the first (top row) and second (bottom row) spatial dynamic mode, visualized by velocity vectors in the cross-sectional plane. (e) Most dominant Fourier mode of the temporal sequence.

\section{Summary, conclusions and outlook}

Three-dimensional flow fields of a transitional water jet have been extracted from experiments by means of time-resolved tomographic particle image velocimetry (TR-TOMO-PIV). The flow fields are characterized by a wide range of spatial and temporal scales, but also by the presence of clearly distinguishable frequencies and wavenumbers. A sequence of forty snapshots in time, each captured with a spatial resolution of $107 \times 62 \times 62$ and three velocity components, has been processed by the dynamic mode decomposition (DMD) - an iterative data-based algorithm for the extraction of dynamically relevant processes from temporally or spatially aligned flow field sequences. In both the temporal and the spatial case, the DMD method isolated coherent structures and their spectral properties and has proven effective in providing a low-dimensional representation of the coherent dynamics.

As experimental data grow larger in dimensionality and complexity, it becomes more important to develop and apply advanced algorithms that are capable of extracting the essential features and dominant processes from the measurements. In particular, the recent availability of tomographic three-dimensional and time-resolved data necessitates these types of algorithms to reduce the richness of three-dimensional flows to a few governing mechanisms. By combining TR-TOMO-PIV data with DMD-analysis, the current article has attempted to integrate state-of-the-art data acquisition techniques with innovative algorithms for flow pattern extraction.

In a future effort, we will further explore the flow features present in the water jet. This study will include longer temporal data sequences as well as measurements further downstream from the jet nozzle. It is hoped that the synthesis of three-dimensional time-resolved data and efficient, data-based, 
iterative algorithms (such as DMD) will give new and valuable insight into complex fluid flow, its principal mechanisms and its inherent spatio-temporal scales.

\section{Bibliography}

[1] Violato, D.; Bryon, K,; Moore, P.; Scarano, F. (2009) Application of Powell's analogy for the prediction of vortex-pairing sound in a low-Mach number jet based on time-resolved planar and tomographic PIV. 16th AIAA/CAES Conference on Aeroacoustics, Stockholm, Sweden (submitted).

[2] Elsinga, G.E.; Scarano, F.; Wieneke, B.; van Oudheusden, B.W. (2006) Tomographic particle image velocimetry. Exp. Fluids, 41, 933-947.

[3] Schmid, P.J. (2009) Dynamic mode decomposition of experimental data. Proc. 8th Int. Symp. on Particle Image Velocimetry, PIV09-0141, Melbourne, Australia.

[4] Schmid, P.J. (2010) Dynamic mode decomposition of numerical and experimental data. J. Fluid Mech., (in press).

[5] Rowley, C.W.; Mezić, I.; Bagheri, S.; Schlatter, P.; Henningson, D.S. (2009) Spectral analysis of nonlinear flows. J. Fluid Mech., 641, 115-127. 